\title{
Estimating fossil ant species richness in Eocene Baltic amber
}

\author{
David Penney and Richard F. Preziosi \\ Acta Palaeontologica Polonica 59 (4), 2014: 927-929 doi:http://dx.doi.org/10.4202/app.00097.2014
}

and provide a unique insight to forest ecosystems of the geological past. Baltic amber has been studied for more than 300 years but despite the large number of described fossil species (ca. 3500 arthropods) and abundance of fossil material, few attempts have been made to try and quantify statistically how well we understand the palaeodiversity of this remarkable Fossil-Lagerstätte. Indeed, diversity estimation is a relatively immature field in palaeontology. Ants (Hymenoptera: Formicidae) are a common component of the amber palaeobiota, with more than 100 described species representing approximately $5 \%$ of all inclusions encountered. Here we apply quantitative statistical species richness estimation techniques to Baltic amber data for the first time. We use species level data from a sample size of 12769 specimens and conclude that around $29 \%$ of the Baltic amber ant fauna has yet to be discovered. The species richness accumulation curve clearly reaches its asymptote at around 9650 specimens, indicating this as the minimum sample size required for a reasonable estimate of species richness for ants alone.

Hence, it is hardly surprising that previous studies concerning so-called "representative" samples of the entire palaeobiota, consisting of at most a few thousand inclusions do not agree with each other.

Nonetheless, we demonstrate that it is possible to apply quantitative techniques to amber derived data and this should be the preferred approach wherever possible, rather than generating qualitative conclusions of little value for comparative purposes.

David Penney [david.penney@manchester.ac.uk] and Richard F.

Preziosi [richard.f.preziosi@manchester.ac.uk], Faculty of Life Sciences, the University of Manchester, Manchester, M13 9PT, UK.

This is an open-access article distributed under the terms of the Creative Commons Attribution License (for details please see creativecommons.org), which permits unrestricted use, distribution, and reproduction in any medium, provided the original author and source are credited. 
Farf Full text (153.8 kB) ।

For's Supplementary file (16.1 kB) 